\title{
XXII. Distribution of the intensity of the radiation from radioactive sources
}

\section{E. Rutherford F.R.S.}

To cite this article: E. Rutherford F.R.S. (1906) XXII. Distribution of the intensity of the radiation from radioactive sources, Philosophical Magazine Series 6, 12:68, 152-158, DOI: $10.1080 / 14786440609463528$

To link to this article: http://dx.doi.org/10.1080/14786440609463528

册 Published online: 16 Apr 2009.

Submit your article to this journal $\sqsubset \pi$

Џ Article views: 2

Q View related articles $\square$

Citing articles: 1 View citing articles 5 
The letter $\mu$ (strictly speaking denoting "molecular conductivity") has been retained, for the sake of simplicity, to represent also "atomic conductivity." The table has been entirely constructed from the data in Landolt-Börnstein's Physikalisch-Chemische Tabellen (latest, 1905, edition). In all cases, the atomic conductivities given are the means of those obtained by all the possible calculations. To calculate the molecular conductivity of any salt, base, or acid, respectively, the equation $\mu v_{\mathrm{M}}+\mu v_{\mathrm{x}}, \mu v_{\mathrm{M}}+\mu v_{\mathrm{OH}}$, or $\mathrm{R} v_{\mathrm{HX}}{ }^{*} \mu v_{\mathrm{H}}+\mu v_{\mathrm{x}}$, should be employed.

East London Technical College, London, E.

XXII. Distribution of the Intensity of the Radiation from Radioactive Sources. By E. RuTHERFond, F.R.S., Macdonald Professor of Physics, McGill University, Montreal *.

[Plate II.]

IN

$\mathrm{N}$ the course of my experiments on the magnetic deflexion of the $a$ rays emitted by the active deposit of radium, a very peculiar photographic effect was observed. The experimental arrangement was similar to that described in a previous paper (Phil. Mag. July 1905). The rays from a wire about $1.5 \mathrm{cms}$. long and $0.5 \mathrm{~mm}$. in diameter, made strongly active by exposure in the radium emanation, passed through a slit and fell on a photographic plate placed some distance above the slit. The whole apparatus was exhausted of air, and a strong magnetic field applied so as to bend the pencil of a rays. With a broad slit, comparable in width to the diameter of the active wire, the photographic trace of the $\alpha$ rays on the plate is shown by a black band with sharply defined edges; and the intensity of the photographic impression is approximately uniform over its cross section. With a very narrow slit, however, the photographic trace of the rays has a very different appearance.

Fig. 1 A, Pl. II., shows a magnified drawing of such a trace. The outside edges of the band are sharply defined, and the photographic impression falls off rapidly from the outside to the centre, but is most intense at the extreme edges. The trace, at first sight, appears as if it consisted of two dark

* Communicated by the Author, having been read before the American Physical Society, April 1906. 
bands with a well-marked white band between them. A photograph taken in a similar way, using an active circular rod $5 \mathrm{mms}$. in diameter, is reproduced in fig. $8, \mathrm{Pl}$. II.

This peculiarity in the trace of the rays could not be due to the magnetic field, for it was observed equally clearly when no magnetic field was acting. The same appearance was also observed when the $\alpha$ rays were passed through an absorbing screen. It was at first thought possible that this effect was due to photographic reversal, since it was first observed when the photographic effects were very intense. Later work, however, showed that the effect was equally marked when the photographic effect was too feeble to admit the possibility of photographic reversal.

In another experiment, a small spbere about $1 \mathrm{~cm}$. in diameter, which was made uniformly active by exposure to the radium emanation, was used as the source of rays. A metal plate was placed about $2 \mathrm{cms}$. above the sphere, and the rays from the sphere passed through a small hole in this plate and fell nearly normally on a photographic plate placed $2 \mathrm{cms}$. above the hole. The impression on the plate corresponded to a pinhole photograph of the active sphere, taken by the $\alpha$ rays emitted from its surface. A drawing of the photograph is shown in fig. $1 \mathrm{~B}, \mathrm{Pl}$. II. The photographic effect is greatest at the extreme edge of the circle, and falls off towards the centre.

This distribution of the inteusity of the radiation for the radioactive sphere and cylinder is very different from that which would be observed if the photographs were taken by the luminous rays from a white-hot sphere or cylinder. In a pinhole image of the sun, for example, we know that the intensity of the radiation is practically uniform over its disk.

These striking differences between the distribution of the intensity of radiation for ordinary light sources and for these radioactive sources receive a simple explanation along the following lines.

In the case of a luminous body like the sun, the intensity of the radiation emitted from any point is not uniform in all directions, but is governed by the well-known cosine law, sometimes known as Lambert's law. This asserts that the intensity of the radiation from any point varies as the cosine of the angle between the normal and the direction of the emitted light. This law of distribution for a self-luminous source is, I believe, usually explained by supposing that the light does not come entirely from the surface, but from a sensible depth of an absorbing layer. 
Let the circle $P$ (fig. 2) represent the cross section of a cylindrical uniformly radiating body. The rays pass through a narrow rectangular slit $\mathrm{CD}$, and fall on a screen SS. The radiation which falls on the central point 0 of the image

Fig. 2.

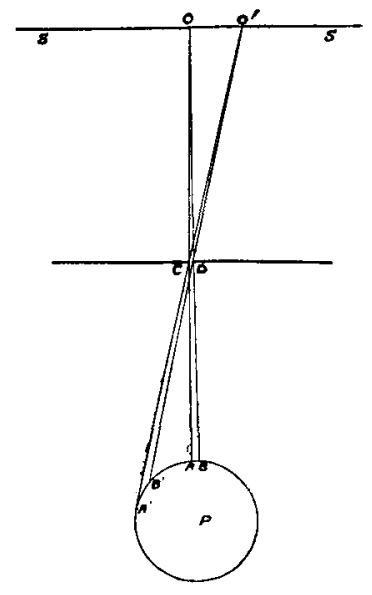

comes from a length' $\mathrm{AB}$ of the circle, which is intersected by lines drawn from $O$ touching the edges of the slit. The radiation which reaches $\mathrm{O}^{\prime}$, a point on the extreme edge of the luminous area, comes from a length $\mathrm{A}^{\prime} \mathrm{B}^{\prime}$ of the circle, which is seen to be considerably greater than the length $A B$.

In the case of a self-luminous body, this increase of radiating surface which supplies light it a point near the edge of the image is exactly compensated for by the cosine law of distribution, and the intensity of the radiation is consequently uniform over the cross section of the image.

In the radicactive case, however, the radiations come from an extremely thin film of radioactive matter, in which the a rays are not appreciably absorbed. There is, in consequence, no cosine law of distribution, but, on an average, the a radiation from any point is equally intense in all directions. We see that, under such conditions, the intensity of the radiations reaching $O$ (disregarding small variations in distance) is proportional to the length $\mathrm{AB}$, and the intensity at $\mathrm{O}^{\prime}$ to the length $A^{\prime} B^{\prime}$. The intensity of the radiation is consequently greatest at the extreme edge of the image, and falls off towards the centre.

If the distances $\mathrm{OC}, \mathrm{CP}$ are very large compared with the radius of the cylinder and the slit very narrow, it can readily 
be shown that the intensity at the extreme edge of the image may be very much greater than that in the centre. This is the experimental case already considered (fig. $1 \mathrm{~A}$, PI II.).

The mathematical theory of the distribution of intensity of the radiation for the case of a radiating sphere or cylinder, when no cosine law applies, can be readily worked out, but it has not been thought necessary to include it here. It suffices to say that the observed distribution of the intensity of the radiation from the radioactive sphere or cylinder receives a simple explanation when we take into account that

(1) the layer of radioactive matter is so thin that the $\alpha$ rays suffer no appreciable absorption in passing through it, $i$. $e$. no cosine law of distribution applies ;

(2) the $\alpha$ particles are, on an average, projected equally in all directions.

The experimental results obtained afford an indirect proof that the $\alpha$ particles are, on an average, projected equally in all directions from a radioactive source.

Onr experience of the distribution of the radiation from luminous sources is so bound up with the cosine law of emission, that we are liable to overlook how different this distribution would be if no cosine law applied. We have seen, for example, that if the radiation from the sun came from a thin non-absorbing superficial laver, the sun would appear to us as a circular disk, the edge of which was marked by a brilliant ring of light, the luminosity falling off rapidly from the edge to the centre.

Consider also another example. If a small plane disk emits light according to the cosine law, the intensity of the radiation at any point some distance from it is greatest when the plane of the disk is normal to the line joining the point and the disk. The intensity of the radiation falls off as the plate is orientated, and is very small when the light is emitted nearly tangentially from the surface. Consider now a similar disk coated with a thin film of radioactive matter, and, in order to avoid the disturbance due to the absorption of the $\alpha$ rays in air, suppose the experiments are made in a vacuum. Since the cosine law does not apply, the intensity of the radiation at some distance from the small plane disk is practically independent of the orientation of the plate, and is nearly the same for normal and tangential emission.

We shall now consider some further experiments which illustrate these points in a striking manner.

A brass rod of square cross section, about $4 \mathrm{cms}$. long, was exposed for some hours to the radium emanation, in order to 
obtain on its surface a thin uniform layer of radioactive matter. After removal, the intensity of the radiation from the rod was examined in various positions, both by optical and photographic methods. In the former, the rod was brought close to a screen of willemite or zinc sulphide.

The results observed are best described by means of a diagram (see fig. 3). ABCD represents the cross section of

Fig. 3.

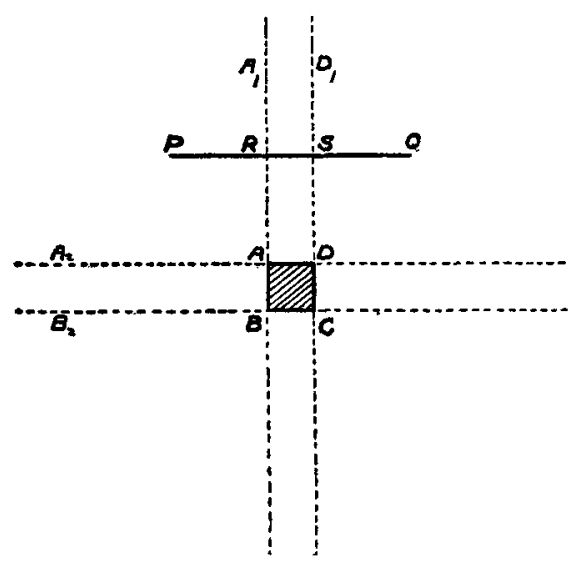

the square rod. The dotted lines represent continuations of the sides of the square. Suppose the phosphorescent screen is placed in the position $\mathrm{PQ}$, parallel to the upper surface of the rod. The experiment at once showed that the part RS within the dotted lines was far less illuminated than the parts $\mathrm{RP}, \mathrm{SQ}$ immediately beyond. This was confirmed by placing a photographic plate in the same position. The intensity of the impression is much less within the region RS than on either side of it. The photographic negative, consequently, has the appearance of a whitish band of width equal to the side of the square on a dark background. The edges of the band are very sharply defined, and there is no gradual transition of intensity from one region to another.

It is, at first sight, somewhat startling that the intensity of the photographic effect is least immediately above and closest to the active surface, but a little consideration shows that such an effect is to be expected. The region RS is acted on only by the rays from the upper surface $\AA \mathrm{D}$ of the rod, while the regions $\mathrm{RP}, \mathrm{SQ}$ are, in addition, acted on by the rays from the sides of the square. It the distance $A R$ is large compared with the length $\mathrm{AB}$, the intensity of the radiation 
just outside the point $R$, due to the side $A B$, is nearly equal to that due to the side AD. Consequently, the intensity of the radiation to the left of the point $R$ is about twice that to the right of $\mathrm{R}$ in the region $\mathrm{RS}$.

This conclusion was confirmed by a slight alteration of the experiment. Two thin plates of metal, which completely absorbed the a rays, were placed in the positions $A R$ and DS. The radiation reaching a point in the region $\mathrm{RP}$ is then due entirely to the rays from the side $A B$. Under such conditions, it was observed that the intensity of the photographic impression in the region RS was very nearly the same as that in the region $R P$ close to $R$.

Some very beautiful optical effects can be produced by a different arrangement of the experiment. The active rod rests on the phosphorescent sereen with its axis normal to the surface. The illuminated area is seen to be divided by four dark bands at right angles to each other, forming a wellmarked cross. The width of these bands is the same throughout their length and equal to the side of the square. The bands correspond to the regions included in fig. 3 between the lines $\mathrm{AA}_{1}$ and $\mathrm{DD}_{1}, \mathrm{AA}_{2}$ and $\mathrm{BB}_{2}, \& \mathrm{c}$. The effects observed are best illustrated by means of the reproduction of an actual photograph shown in fig. 4, Pl. II. The active rod rested normally on the photographic plate, as in the case of the experiment with the screen.

The explanation of these bands, where the luminosity is less than in the region immediately outside them, is obvious from what has already been stated.

In most of the experiments, the rod was charged negativeily, then exposed in the emanation vessel, in order to concentrate the active deposit upon it. The active deposit was consequently not uniformly distributed, being in somewhat greater amount at the edges of the rod. This lack of uniformity in the distribution had, however, no sensible effect on the main features of the photographs, for equally clear effects were obtained when no electric field was acting during the exposure of the rod to the emanation. With an active rod clear photographs of this character can be obtained in a few minutes.

It was thought of interest to examine the distribution of the radiation in two other cases. In one experiment, the cross section of the rod was an equilateral triangle of $6.5 \mathrm{mms}$. side, and in the other a regular hexagon of 2.75 mms. side. The rods were placed normally on the plate, and the photographs obtained are shown in figs. 5 and 6, Pl. II.

'The explanation of the results obtained is clearly seen from 
the dingrams shown in fig. 7. $\mathrm{ABC}$ is the cross section of the triangular rod. The regions $a, b$, and $c$, bounded by the continuations of the sides of the triangles, are clearly shown in the photograph, since the radiation outside these triangular areas is due to one side alone.

Fig. 7.

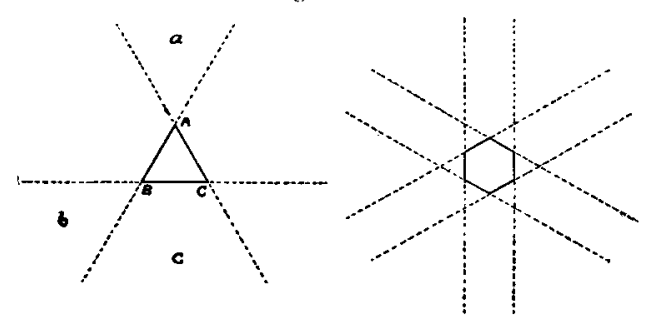

The photograph obtained with the hexagonal rod presents several points of interest. A six-armed cross isiclearly seen as well as the outline of the six small equilateral triangles obtained by the continuation of alternate sides of the hexagon. The explanation of this six-armed cross and the smaller equilateral triangles is clear from the diagram in fig. 7, where the full lines represent the hexagon, and the dotted lines the boundaries of the arms of the cross and of the triangles.

A sudden change of intensity occurs at every point where the tangential radiation from another side of the rod adds its effect.

Experimental examples of this kind can easily be multiplied, but sufficient have been given to illustrate the striking variations of the intensity of the radiation round a radiating body when no cosine law of distribution applies. There is no doubt that similar effects could be obtained if the $\beta$ instead of the a rays were used as a source of rays. The lines of demarcation of the shadows would not, however, be so marked as in the case of the $\alpha$ rays, on account of the considerable penetrating power of the $\beta$ rays.

Theoretically, the distribution should be quite different if the radiation comes from a thick instead of a thin layer of radionctive matter. It is diffeult to obtain such radiouctive sources in definite geometric form, but we should expect that the distribution for a thick layer should approximate closely to that observed for light sources where the cosine law of emission applies.

I am indebted to my colleague, Professor Cox, for his assistance in explaining these effects.

McGill University, Montreal, June 5, 1906. 
FIG. 3 в.

FIG. 3 A.
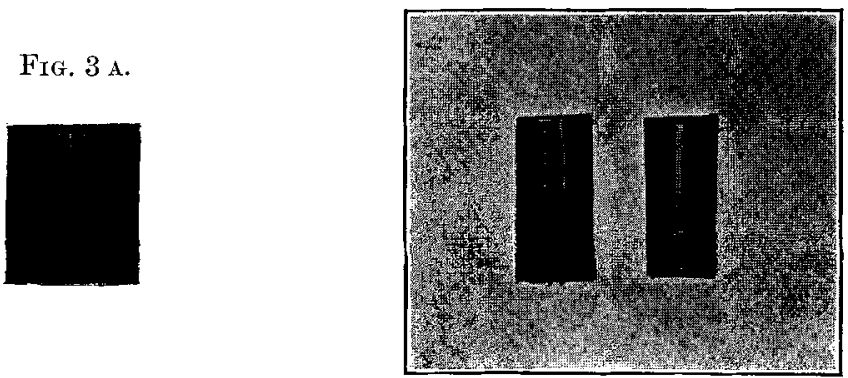

FIf. 1

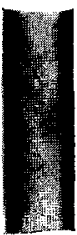

A.

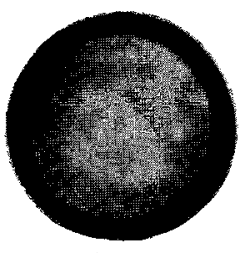

$B$. 
Phil. Mag. Ser. 6, Vol. 12, PI. II.

Figr. 4.

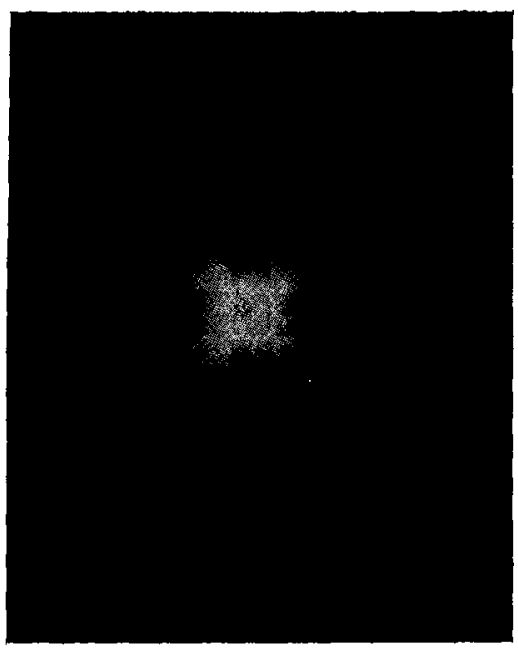

Fig. 5.

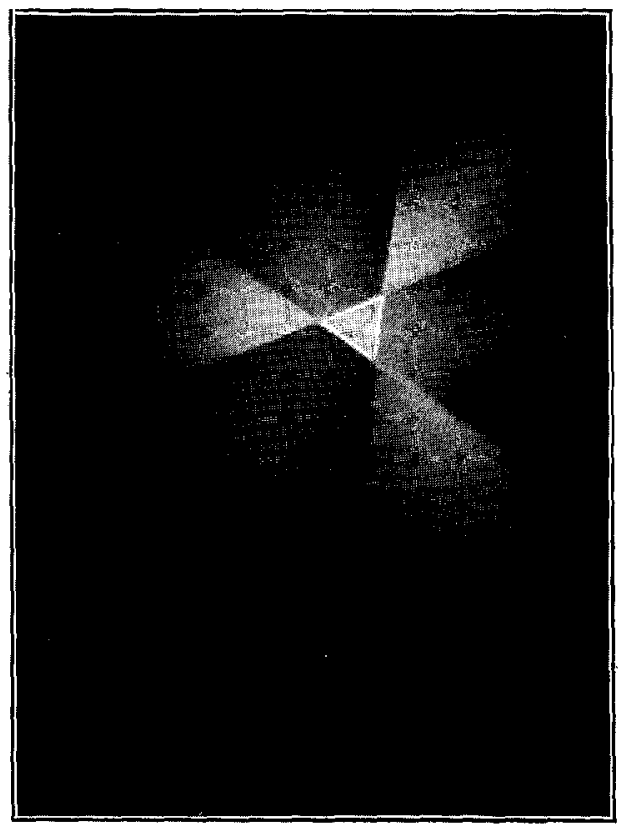

Fig. 6.

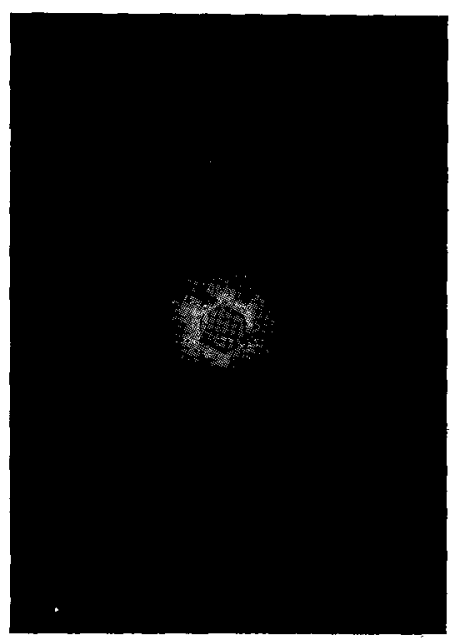

Fig. 8.

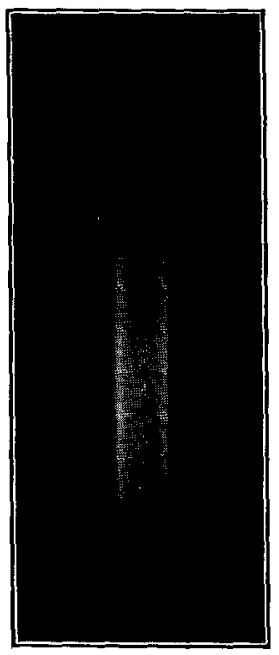

